\title{
Influence du génotype et du milieu sur les tests d'appréciation de la valeur d'utilisation du blé tendre (Triticum aestivum em. Thell.) dans les industries de cuisson
}

\author{
Michel ROUSSET, Eugène TRIBOI $\left({ }^{*}\right)$, Gérard BRANLARD \& Bernard GODON $\left({ }^{* *}\right)$ \\ I.N.R.A., Station d'Amélioration des Plantes, F 63039 Clermont-Ferrand Cedex. \\ (*) I.N.R.A., Station d'Agronomie, 12, avenue du Brézet, F 63039 Clermont-Ferrand Cedex. \\ (**) I.N.R.A., Laboratoire de Biochimie et Technologie des Protéines, La Géraudière, F 44072 Nantes Cedex.
}

Le but des expérimentations rapportées dans cet article est de mieux expliciter la nature des relations entre différents tests de la valeur d'utilisation du blé tendre et ainsi de mieux comprendre l'influence des caractéristiques des échantillons d'étude. Les tests étudiés sont : teneur en protéines totales, indice Pelshenke, indice de sédimentation de Zeleny, indice de sédimentation en milieu S.D.S., essai de l'alvéographe Chopin, p. 100 d'hydratation au farinographe Brabender, temps de chute de Hagberg, essai de panification CNERNA, essai de cuisson miniaturisé (Godon), essai de microcuisson (Bourdet). Les échantillons d'étude proviennent de deux grands types d'essais:

- l'un consiste en l'expérimentation de 2 cultivars "Courtot» et "Hardi » auxquels sont appliquées 16 conditions de culture différentes avec des alimentations azotées très variées en quantité et en qualité. Ces conditions se sont traduites par des rendements et des valeurs d'utilisation très diversifiés pour un génotype donné (variation « environnementale »).

- l'autre est l'expérimentation multilocale d'une série de génotypes diversifiés pour les caractéristiques de valeur d'utilisation ; les variations observées sur ces échantillons recouvrant à la fois une variation génétique et une variation " environnementale».

Suivant que l'on s'adresse à une variation génétique ou " environnementale ", les relations entre les tests, appréciées par les coefficients de corrélation, sont tout à fait différentes. Dans le cas d'une variation « environnementale ", les fluctuations des tests, pour un génotype donné, sont souvent directement reliées à la variation quantitative des protéines. Cependant, les relations unissant les différents tests à la concentration des protéines dans le grain sont différentes. Par contre, dans l'essai « inter-stations » où, dans chaque lieu, on a affaire à une variation essentiellement génétique, les variations des tests seraient plutôt reliées à la variation qualitative des protéines (proportions relatives des différentes fractions protéiques).

A la lumière de ces résultats, les auteurs préconisent le plus grand soin dans le choix et la description des échantillons retenus dans l'étude de nouveaux tests et des bases biochimiques de la valeur d'utilisation du grain de blé.

Mots clés additionnels : Corrélations environnementales, corrélations phénotypiques, tests technologiques. evaluated in tests of baking quality.

The purpose of the experiments described in this paper was to understand the nature of the relationships observed between different tests of baking quality and then to obtain a better understanding of the influence of the characteristics of the samples under study on the level of correlation observed between the tests. The following tests were compared in our experiments: crude protein content, Pelshenke test, Zeleny sedimentation value, SDS sedimentation value, Chopin alveograph, percentage hydration with Brabender farinograph, Hagberg falling number, CNERNA breadmaking assay, micro cooking tests of Godon and Bourdet. The samples studied came from two main experiments : 1) 2 cultivars grown in one year at one location under 16 growing conditions differing qualitatively and quantitatively in nitrogen nutrition. Under such conditions one observed fairly high variation in yield and in baking quality for the same genotype (environmental variation) ; 2) study of a series of genotypes highly diversified for their baking quality, grown in a multilocation trial. The variations observed in the harvested samples were due partly to genetic variation and partly to environmental variation. Phenotypic correlation coefficients were calculated within each location and for all the locations (between-cultivar means calculated over all locations). Depending on the origin of the variation (genotypic or environmental) the relationships observed between quality tests were completely different. In the 'agronomy trial', the environmental variation in the tests for a given genotype was very 
often in direct relation with the quantitative variation in proteins. However, depending on the test considered, the pattern of variation in relation to protein content could be very different. In contrast, in the « interstation trial ", we had to deal mainly with genotypic variation within each location, variation in the quality tests was more related to qualitative variation in proteins (relative proportions of the protein fractions). From these results, the authors propose that great care should be taken in choosing and describing the samples analyzed in studies on new technological tests and on the biochemical basis of the baking quality of wheat grain.

Additional key words : Environmental correlations, phenotypical correlations, technological tests.

\section{INTRODUCTION}

L'appréciation de la valeur d'utilisation des génotypes en cours de sélection et de lots de grains au moment des transactions se fait par le recours à différents tests qui apportent parfois des informations discordantes. Ainsi BOURDET et al. (1971) signalent des corrélations contradictoires entre les différents critères d'appréciation des aptitudes boulangères variétales. Dans leur conclusion, il est reconnu que les protéines peuvent jouer un rôle par leur variation en quantité et en qualité. Le bilan de ces comparaisons est en faveur de l'indice de sédimentation de Zeleny et de certains aspects de l'alvéogramme Chopin et nettement en défaveur de l'indice Pelshenke, comme critères prédicteurs du volume du pain dans des essais de panification. Cependant, une analyse bibliographique révèle que, bien souvent, les auteurs travaillent sur une variation valablement décrite (gamme de variation, moyenne, variance...) mais qui peut provenir, suivant les cas :

- du génotype : par exemple, une série de génotypes bien définis (variétés) sont cultivés dans des conditions de milieu bien précises (lieu, année...) ;

- de l'environnement : par exemple, un génotype particulier est cultivé dans des environnements très diversifiés (lieux, années, techniques culturales...) ; - de ces 2 facteurs conjugués génotype et environnement : il est fréquent par exemple que les échantillons soient constitués de séries variétales cultivées différentes années.

Il arrive même que, dans certains cas, la provenance des échantillons d'étude ne soit pas précisée.

Notre souci d'améliorer l'efficacité de la sélection pour la valeur d'utilisation nous a conduits à tenter de caractériser la plupart des tests disponibles en France pour leur aptitude à apprécier ce que ROUSSET \& LOISEL (1984) appellent la valeur génotypique d'utilisation qui intègre la "valeur potentielle d'utilisation" et une " amplitude de variation » de la valeur d'utilisation dans un ensemble de milieux diversifiés.

Le but de cet article est d'étudier la variation des tests d'appréciation de la valeur d'utilisation et de montrer comment les relations entre ces tests peuvent être modifiées en fonction de l'origine de la variation.

\section{MATÉRIEL ET MÉTHODES}

Les échantillons analysés proviennent de 2 grands types d'expérimentations :

- Essai "Agronomie », dans lequel on a étudié, en un seul lieu, le comportement de 2 génotypes dans un grand nombre de milieux nutritionnels très différenciés.

- Essai "Inter-stations » dans lequel une série de génotypes variés a été expérimentée dans différents lieux.

\section{A. Essai Agronomie}

Conduit en 1977 à la Station d'Agronomie de Clermont-Ferrand.

Deux cultivars bien adaptés à ce milieu ont été retenus : " Hardi » et " Courtot ».

« Hardi » possède un développement de type hiver, une taille classique d'environ $1 \mathrm{~m}$; son épiaison est demi-précoce.

"Courtot " est un blé très alternatif, précoce, portant les 2 gènes de demi-nanisme rht 1 et rht 2 de «Norin $10 »$.

Tous les 2 possèdent une bonne valeur d'utilisation qui les place en classe $\mathrm{B}$ des blés panifiables (cf. règlement technique du Comité Technique Permanent de la Sélection $=$ CTPS). Leur rendement en Limagne est assez élevé. Le comportement des 2 cultivars a été testé en 1977 dans le dispositif de longue durée mis en place en 1949 et destiné à comparer différents systèmes culturaux en " terre noire " de Limagne.

Ce dispositif étant décrit par ailleurs (GACHON, 1973, 1975), nous ne faisons, dans le tableau 1, qu'un bref rappel des paramètres étudiés.

Les rendements obtenus ont été moyens mais fortement différenciés en fonction du traitement. Les conditions nutritionnelles ont produit une variation, d'une part, du rendement, de 22 à $67 \mathrm{q} /$ ha de matière sèche, et, d'autre part, de la teneur en protéines, de 9 à 14 p. 100 (fig. 1).

\section{B. Essais inter-stations}

Les lignées avancées des laboratoires de sélection du blé tendre du Département de Génétique et d'Amélioration des plantes sont testées chaque année dans des essais multilocaux. On limite les interactions entre génotypes dues à des différences de taille trop importantes en homogénéisant les hauteurs dans 2 essais séparés " paille courte " et " paille longue ». En 197677,10 et 13 génotypes figuraient, respectivement, dans chacun des essais.

Pour l'ensemble des tests réalisés, les analyses ont porté sur un échantillon variétal moyen (mélange de répétitions) de chacun des lieux (tabl. 6). 
TABLEAU 1

Facteurs étudiés dans l'essai « Agronomie 》 - Crouelle $C_{I}$.

Factors studied in the "agronomy" trial in which local agronomic conditions were varied - Crouelle $C_{1}$.

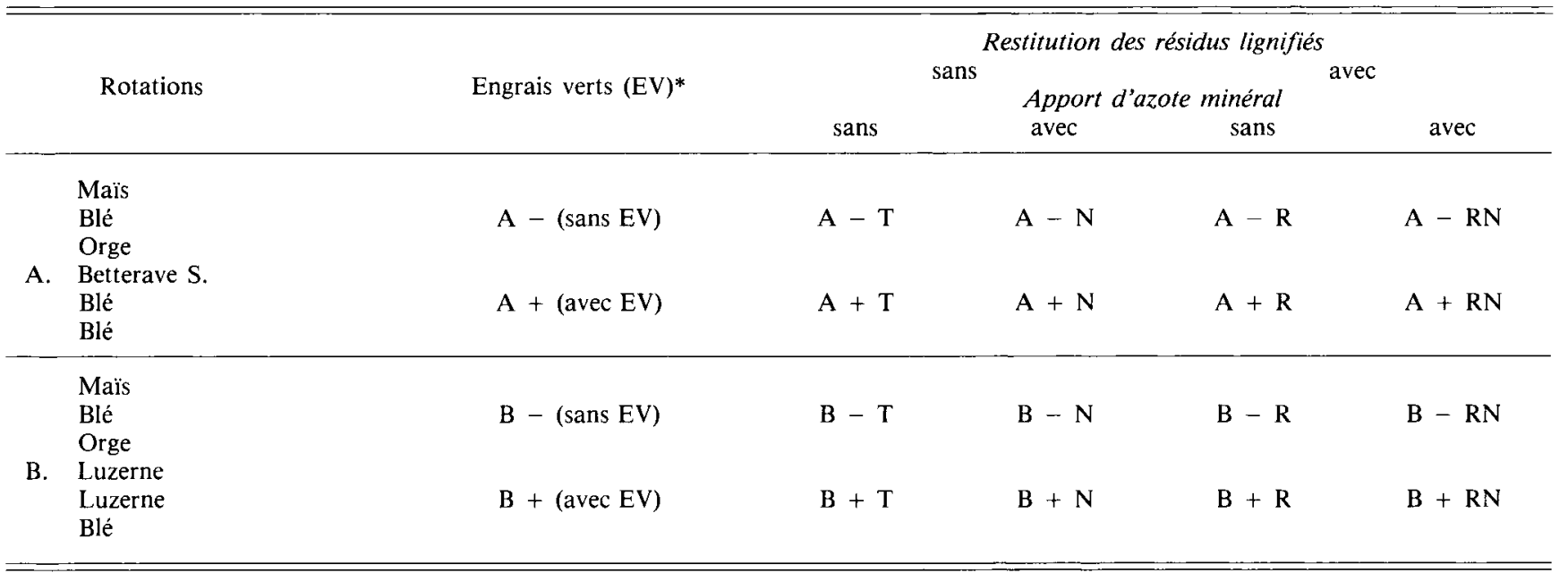

* Chaque fois que cela est possible une vesce est cultivée en dérobé derrière céréales. De plus, sont incorporés au sol les verts de betteraves en $\mathrm{A}+$ et le dernier cycle de luzerne en $\mathrm{B}+$.

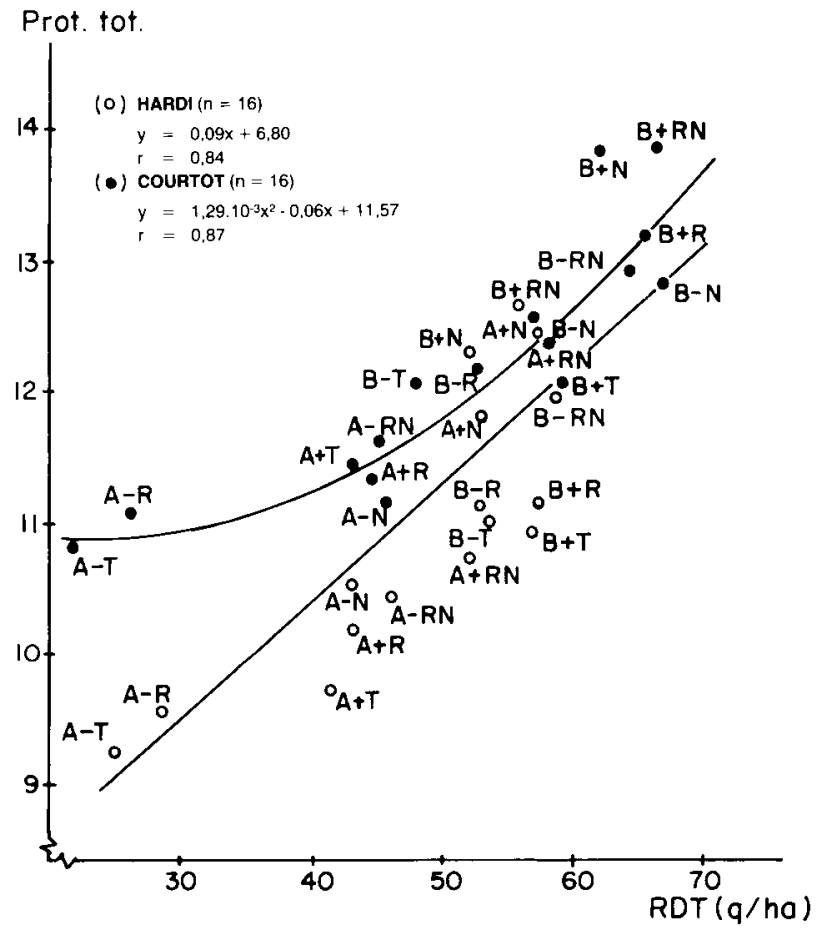

Figure 1

Relations entre la teneur en protéines totales et le rendement en grain.

Relationship between crude protein content and grain yield.

\section{Méthodes}

\section{Analyses technologiques}

Le tableau 2 présente les différents tests réalisés dans chacun des essais, le mode d'expression des résultats et les unités choisies.

Certains tests n'ont pas été réalisés sur tous les essais, les résultats les concernant seront néanmoins présentés mais, bien entendu, la comparaison de leur comportement entre les 2 grands types d'expérimentation ne pourra être faite.

Pour la description des tests et la bibliographie s'y rapportant, on peut se référer à l'article de ROUSSET \& LOISEL (1984) ou encore au guide pratique de la qualité des blés (MAUZE et al., (1972). Seul le test de sédimentation en milieu SDS que nous avons modifié mérite une description détaillée.

Ce test a été proposé à l'origine par AXFORD et al. $(1978,1979)$; on utilise les mêmes réactifs aux mêmes concentrations que dans la méthode originale : $20 \mathrm{~g}$ de grains sont broyés (mouture entière) et $6 \mathrm{~g}$ de mouture sont introduits dans chaque cylindre gradué. On utilise les cylindres de $100 \mathrm{ml} \mathrm{du}$ test de Zeleny.

Les modifications essentielles que nous avons apportées à la méthode originale concernent les points suivants :
Température de réalisation du test

Moulin utilisé pour préparer la mouture d'analyse

Agitation des cylindres

\begin{tabular}{c} 
Méthode originale \\
\hline bain-marie à $22{ }^{\circ} \mathrm{C}$ \\
Tecator Cyclotec Mill \\
(Udy Cyclone Mill) \\
Manuelle
\end{tabular}


TABLEAU 2

Les tests de valeur d'utilisation réalisés dans chacun des essais. Baking quality tests done for each trial.

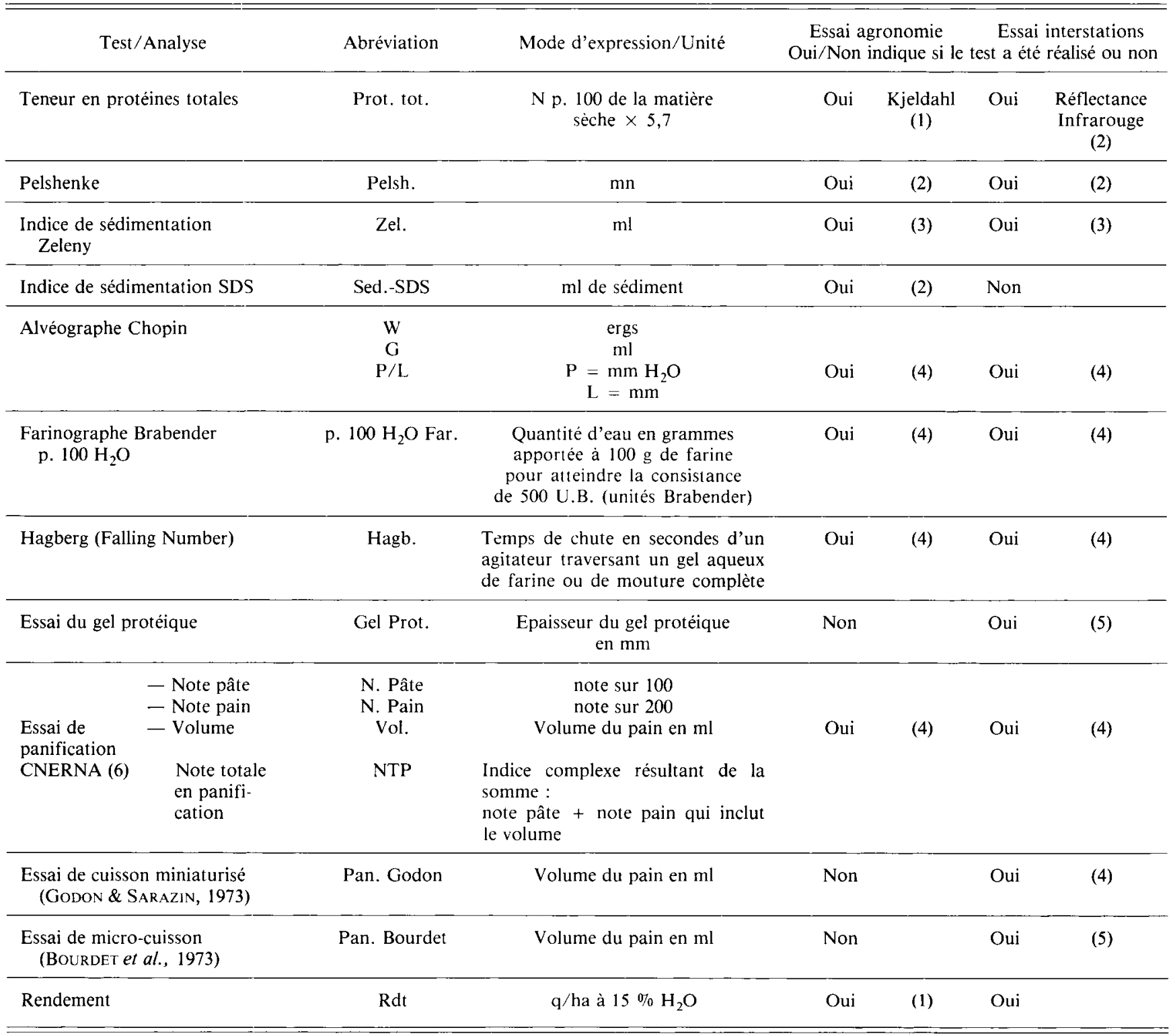

Laboratoire d'analyse :

(1) : Station d'Agronomie de Clermont-Ferrand (département d'Agronomie).

(2) : Laboratoire du blé de la Station d'Amélioration des Plantes de Clermont-Ferrand.

(3) : Laboratoire du blé de la Station d'Amélioration des Plantes de Dijon.

(4) : Laboratoire de Biochimie et Technologie des Protéines du Centre de Recherches de Technologie Agro-Alimentaire de Nantes.

(5) : Laboratoire de Technologie des Céréales du Centre de Recherches Agronomiques de Montpellier.

(6) : CNERNA : Centre National de Coordination des Etudes et Recherches sur la Nutrition et l'Alimentation.

Une fois les échantillons de mouture introduits dans les cylindres, le mode opératoire est le suivant :

- ajouter $50 \mathrm{ml}$ d'eau désionisée,

- agiter vigoureusement pendant $5 \mathrm{~s}$ en maintenant le cylindre dans la position horizontale (cela correspond à 12 agitations de droite à gauche),

- placer le cylindre dans le cadre de l'agitateur mécanique Zeleny, agiter $5 \mathrm{mn}$,

- retirer le cylindre de l'agitateur et retourner 4 fois pour mélanger,

- ajouter $50 \mathrm{ml}$ du réactif SDS - acide lactique porté à $22{ }^{\circ} \mathrm{C}$,

- retourner le cylindre 4 fois pour mélanger,
- placer le cylindre sur l'agitateur Zeleny, agiter 5 $\mathrm{mn}$,

- retirer le cylindre de l'agitateur et mettre en position verticale,

- laisser reposer $20 \mathrm{mn}$ avant de lire le volume de dépôt.

\section{Analyses statistiques}

L'analyse de variance (blocs Fisher) appliquée aux résultats inter-stations, en considérant les lieux comme des répétitions et les génotypes comme " traitement principal », va nous permettre d'évaluer le rapport $\frac{\sigma_{G}^{2}}{\sigma_{L}^{2}}$ 
dont nous verrons la signification plus loin. On calcule ce rapport à partir des termes du tableau d'analyse de la variance de la manière suivante :

$$
\begin{aligned}
\sigma_{\mathrm{G}}^{2} & =\frac{\mathrm{CMG}-\mathrm{CMR}}{\text { nombre de lieux }} \\
\sigma_{\mathrm{L}}^{2} & =\frac{\mathrm{CML}-\mathrm{CMR}}{\text { nombre de génotypes }}
\end{aligned}
$$

avec $\mathrm{CMG}=$ Carré Moyen Génotypes

$\mathrm{CML}=$ Carré Moyen Lieux

$\mathrm{CMR}=$ Carré Moyen « Résiduelle ».

Ce calcul est possible en admettant que l'on se situe dans un modèle à effets aléatoires. On suppose que les génotypes sont tirés au hasard dans une population constituée de toutes les lignées qu'il est théoriquement possible d'obtenir dans un programme de sélection. Les lieux sont tirés d'une population constituée de tous les milieux que l'on peut théoriquement rencontrer sur l'aire potentielle de culture des lignées sélectionnées.
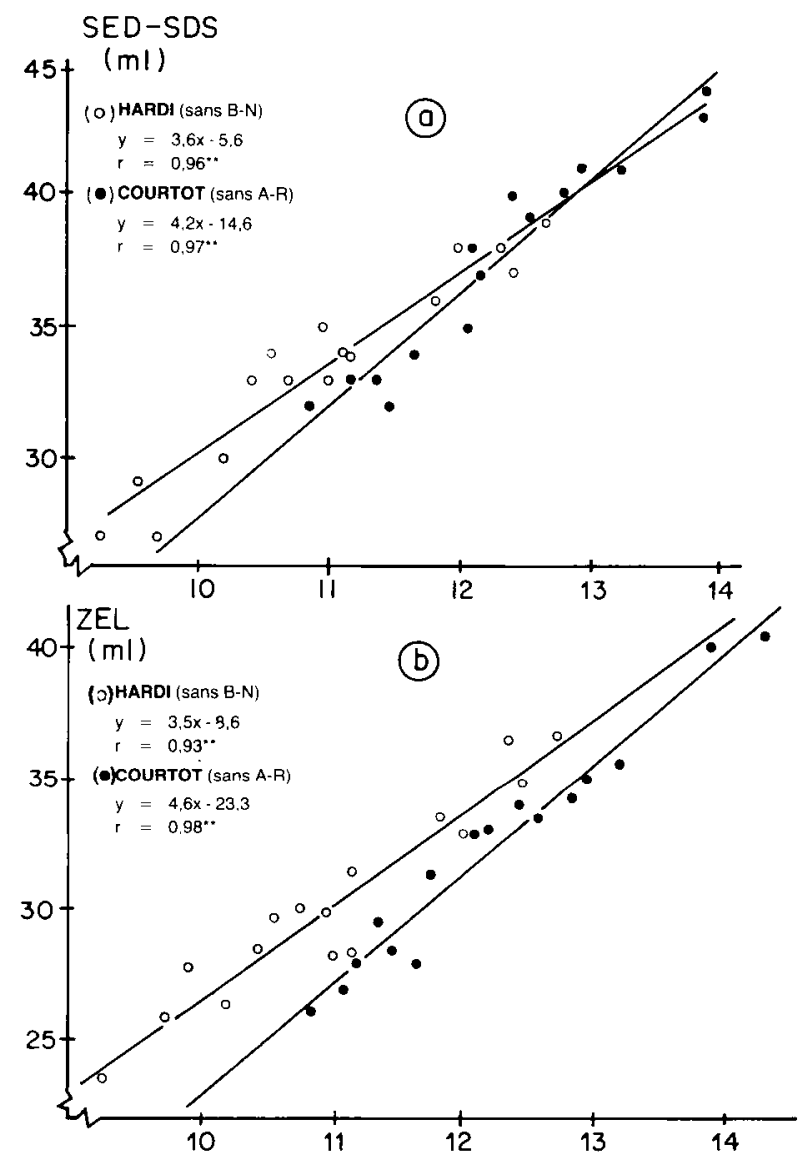

Figure 2

$a$-Relations entre le test de sédimentation SDS et la teneur en protéines totales.

Relationship between SDS sedimentation test and crude protein content.

$b-$ Relations entre le test de sédimentation Zeleny et la teneur en protéines totales.

Relationship between Zeleny sedimentation test and crude protein content.
On admettra que, dans les essais inter-stations, ces hypothèses sont vérifiées.

\section{RÉSULTATS}

\section{A. Essai Agronomie}

Dans les figures 2 et 3 nous présentons les relations mises en évidence entre chacun des tests, d'une part, la teneur en protéines totales, d'autre part. Du fait de quantités insuffisantes, les échantillons B-N pour « Hardi » et A-R pour «Courtot» n'ont pu subir l'ensemble des analyses et ont été éliminés des calculs.

On observe ainsi différents types de comportements que l'on peut classer dans les catégories suivantes:

\section{Régression linéaire du test sur la teneur en pro- téines totales}

Dans le cas des indices de sédimentation SDS (fig. 2a) et Zeleny (fig. 2b) et pour le volume du pain dans

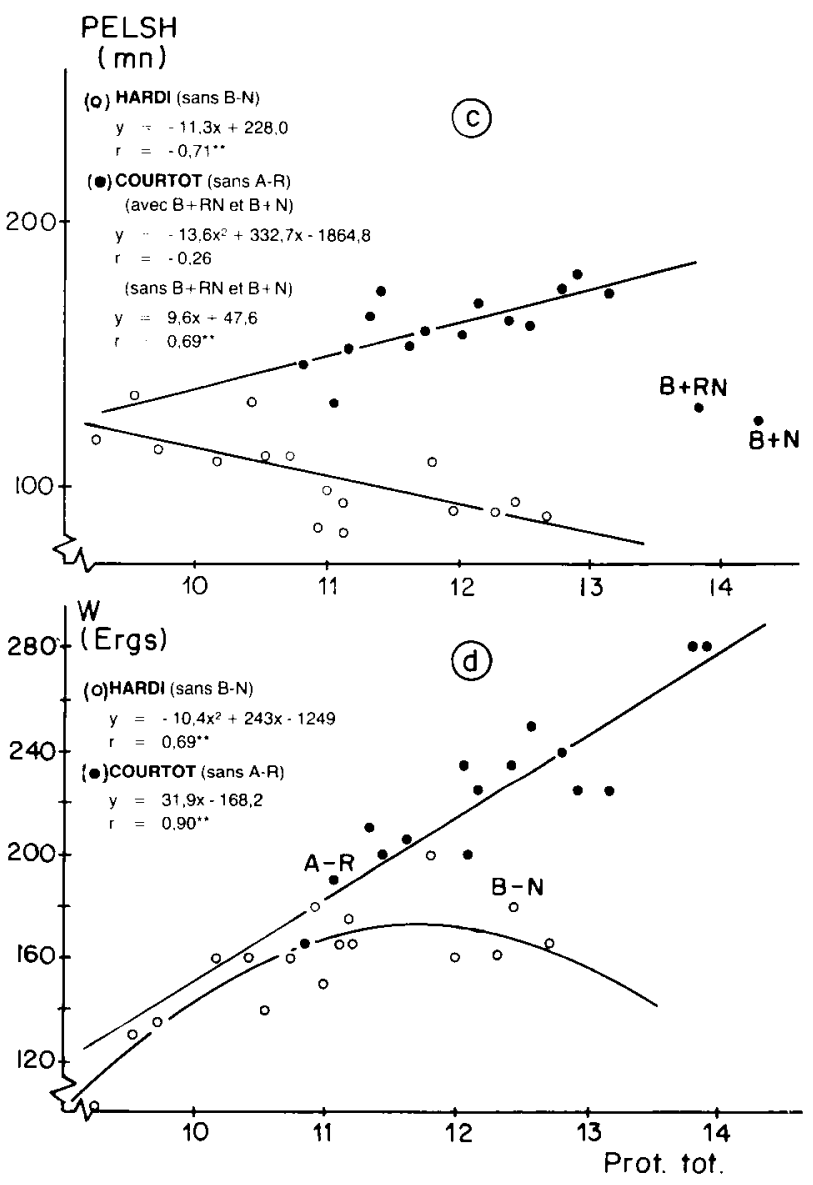

c-Relations entre le test de Pelshenke et la teneur en protéines totales.

Relationship between Pelshenke test and crude protein content.

$d$-Relations entre le test $W$ de l'alvéographe et la teneur en protéines totales.

Relationship between $W$ alveograph test and crude protein content. 

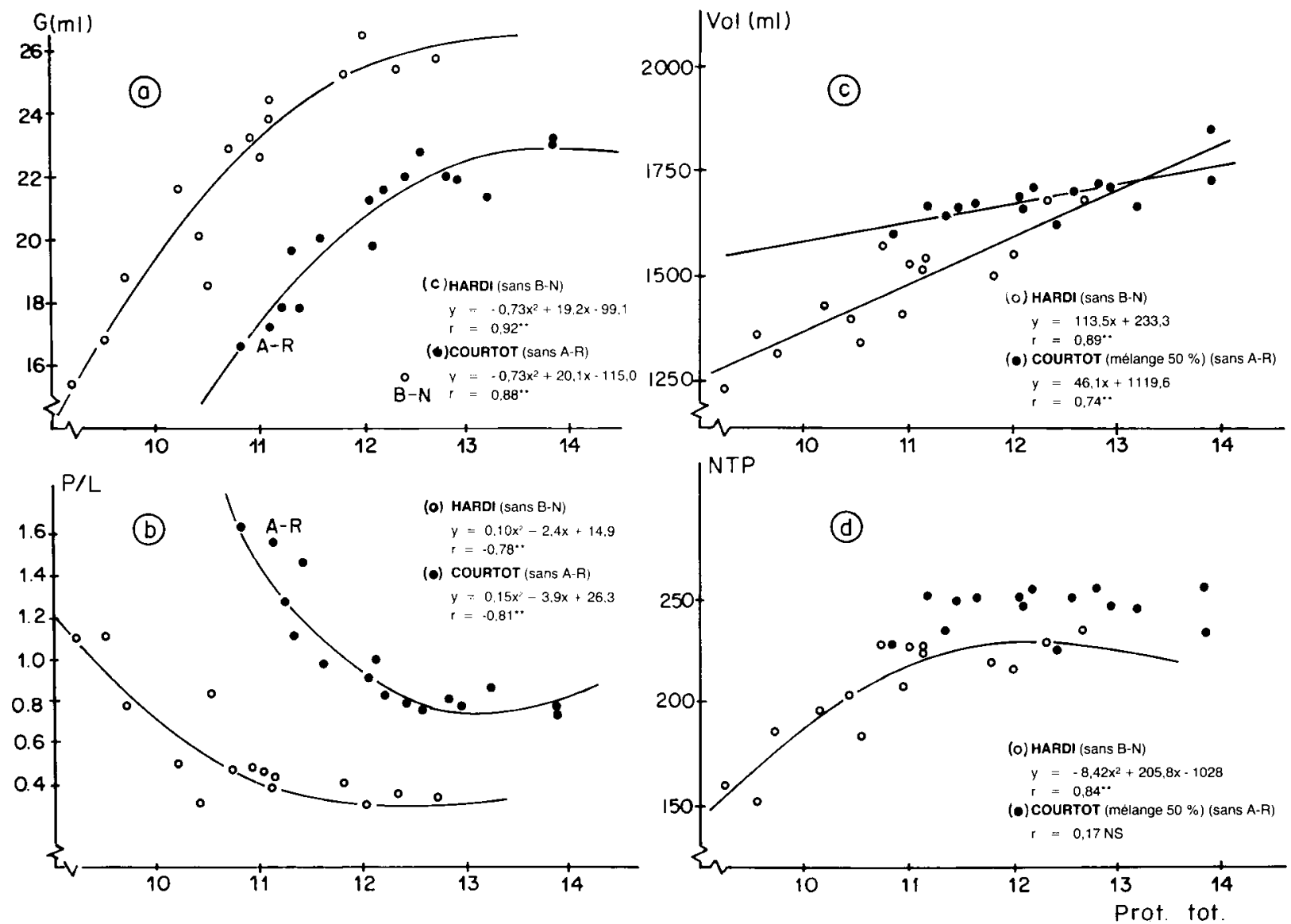

Figure 3

$a-$ Relations entre le test $G$ de l'alvéographe et la teneur en protéines totales.

Relationship between $G$ alveograph test and crude protein content. $b-$ Relations entre le test $P / L$ de l'alvéographe et la teneur en protéines totales.

Relationship between $P / L$ alveograph test and crude protein content.

$c$ - Relations entre le test Volume du pain de l'essai de panification CNERNA et la teneur en protéines totales.

Relationship between Bread Volume (CNERNA breadmaking test) and crude protein content.

$d$ - Relations entre le test Note totale en panification de l'essai CNERNA et la teneur en protéines totales.

Relationship between Total Breadmaking value (CNERNA test) and crude protein content.

l'essai CNERNA (fig. 3c), les régressions linéaires sont étroites et de même signe $(+)$ pour les 2 génotypes étudiés.

En ce qui concerne le W de l'alvéographe Chopin (fig. 2d), il n'apparaît une telle régression que pour «Courtot».

\section{Régression du second degré du test sur la teneur en protéines totales}

On observe des régressions du second degré dans le cas du W (fig. 2d) et de NTP (fig. 3d) pour «Hardi », du G (fig. 3a) et du P/L (fig. 3b) de l'alvéographe pour les 2 cultivars. Les coefficients de corrélation sont hautement significatifs et de même signe, positif, dans tous les cas à l'exception du rapport $\mathrm{P} / \mathrm{L}$.

\section{Absence de liaison nette entre le test et la teneur en protéines totales}

Dans le cas de l'indice Pelshenke, malgré un coefficient de corrélation négatif hautement significatif entre les résultats du test et la teneur en protéines

totales pour " Hardi », les données des 2 cultivars sont contradictoires et on ne peut mettre en évidence une tendance claire quant à l'influence de la teneur en protéines totales sur l'expression du test.

Les régressions linéaires positives entre résultats des tests et teneur en protéines totales indiquent une influence directe de la variation quantitative des protéines sur les caractéristiques mesurées tandis que, dans le cas des régressions du second degré, la seule variation quantitative des protéines ne suffit plus à expliquer la variation des tests considérés. L'explication est alors, sans doute, à rechercher dans un changement de la qualité des protéines qui doit suivre l'évolution de la quantité totale des protéines. Cette hypothèse est à rapprocher des résultats obtenus sur certains des échantillons par BRANLARD \& TRIBOI (1983). Il s'agit des modifications des proportions relatives des fractions protéiques entre elles ou avec d'autres constituants (amidon, lipides, etc.).

Dans ce sens, chez le cultivar "Courtot" les traitements $B+R N$ et $B+N$ en présence d'engrais verts sont particulièrement intéressants. Dans la comparaison entre le test Pelshenke et la quantité totale des protéi- 
nes du grain, ces 2 valeurs s'écartent de la régression linéaire générale. Ce comportement « anormal » peut s'expliquer par le fait que ces 2 traitements ont, d'une part, le poids du grain le plus faible, environ $30 \mathrm{mg}$ par rapport à 32-38 mg dans les autres traitements, et, d'autre part, la teneur en protéines la plus élevée, au moins de $0,6 \%(0,1 \% \mathrm{~N})$. Dans ces grains qu'on peut considérer « échaudés », la composition biochimique est affectée, d'où un comportement particulier mis en évidence principalement par le test Pelshenke.

Les résultats de l'étude des corrélations entre tests (tabl. 3) susceptibles de nous informer sur leur signification technologique sont, en conséquence, à considérer avec une grande prudence. Pour clarifier la discussion nous ne présentons que les résultats des corrélations avec le $\mathrm{W}$ parallèlement aux corrélations avec la teneur en protéines totales.

La confrontation de ces 2 séries de coefficients de corrélation, en particulier dans le cas de "Courtot» pour lequel le $\mathrm{W}$ de l'alvéographe apparaît étroitement lié à la teneur en protéines totales, les coefficients de corrélation entre le $\mathrm{W}$ et les différents tests suivent très fidèlement les coefficients de corrélation de ces mêmes tests avec la teneur en protéines totales.

Ainsi, le niveau élevé de certaines liaisons (tabl. 3), comme W-ZEL ou W-SED.SDS, doit être considéré principalement comme la conséquence d'une même cause : la variation quantitative des protéines.

\section{Remarque :}

La simple prise en compte du niveau des corrélations observées entre les différents tests avec un test de référence tel que le $\mathrm{W}$ de l'alvéographe CHOPIN, à partir d'échantillons obtenus dans des conditions analogues à celles de l'essai « Agronomie ", pourrait conduire à des conclusions qui se révèleront plus loin erronées concernant le choix de "bons tests", tels que les indices de sédimentation Zeleny ou SDS ou, au contraire, le rejet de «mauvais tests », tels que le Pelshenke (pour l'appréciation de la valeur d'utilisation de lots de grains ou de génotypes).

\section{B. Essais Inter-Stations}

Dans les essais inter-stations, la nature de la variation est fondamentalement différente de celle observée dans le cas de l'essai «Agronomie ». Dans l'étude conduite ici, nous tentons de caractériser, d'une part, la variation due aux génotypes, d'autre part, la variation due à l'environnement c'est-à-dire aux différents lieux de culture. L'analyse de variance consistant à considérer les différents lieux comme des répétitions ( ( blocs $»)$ et les génotypes comme traitement principal ne nous permet pas de prendre en compte le facteur interaction «génotype $\times$ lieu ».

Le tableau 4 présente la variation des différents tests d'appréciation de la valeur d'utilisation et les composantes de cette variation. On constate que, dans la plupart des cas, l'étendue de variation des « moyennes génotypes » (col. 4) est plus large que celle des « moyennes lieux » (col. 5). Les nombres de classes de "qualité » (col. 7) et les coefficients de variation (col. 8) nous renseignent sur l'importance de l'erreur propre à chacun des tests et sur l'aptitude de ceux-ci à différencier les génotypes lorsqu'on applique un crible de sélection. Il est à remarquer que l'erreur calculée dans l'analyse de variance inclut, en plus de l'erreur «sensu stricto », la variation due aux interactions génotype $\times$ lieu. Enfin le rapport $\frac{\sigma_{G}^{2}}{\sigma_{L}^{2}}$ (col. 9), qui permet une approche de l'importance relative de la variation due aux génotypes et de celle due aux milieux, révèle les possibilités de prédiction de la valeur d'un lot de grains par la seule connaissance de sa constitution génétique.

\section{Remarque :}

Un rapport $\frac{\sigma_{\mathrm{G}}^{2}}{\sigma_{\mathrm{L}}^{2}}$ élevé peut résulter d'une valeur $\sigma_{\mathrm{G}}^{2}$ élevée, c'est-à-dire d'une large variabilité génétique, et/ou d'une valeur $\sigma_{\mathrm{L}}^{2}$ faible, c'est-à-dire d'une faible influence du milieu sur l'expression du caractère.

TABLEAU 3

Essai Agronomie : Coefficients de corrélation entre la teneur en protéines totales, le $W$ de l'alvéographe, d'une part, les différents tests d'appréciation de la valeur d'utilisation, d'autre part (I).

"Agronomy" trial : correlation coefficients between crude protein content and $W$ alveograph on one hand and the different baking quality tests on the other.

\begin{tabular}{|c|c|c|c|c|c|c|c|c|c|c|c|c|}
\hline \multirow{4}{*}{ Hardi } & \multirow[b]{3}{*}{ Prot. tot. } & \multirow{3}{*}{$\begin{array}{c}\text { Prot. tot. } \\
1 \\
\end{array}$} & \multirow{3}{*}{$\begin{array}{c}\text { Zel. } \\
0,93^{* *}\end{array}$} & \multirow{3}{*}{$\begin{array}{c}\text { Sed. SDS } \\
0,96^{* *}\end{array}$} & \multirow{3}{*}{$\begin{array}{l}\text { Pelsh. } \\
-0,71^{* *}\end{array}$} & \multirow{3}{*}{$\begin{array}{c}\mathrm{W} \\
0,69^{* *}\end{array}$} & \multirow{3}{*}{$\frac{G}{0,92 * *}$} & \multirow{3}{*}{$\begin{array}{c}\mathrm{P} / \mathrm{L} \\
-0,78^{* *}\end{array}$} & \multirow{3}{*}{$\begin{array}{c}\text { Hagb. } \\
-0,02\end{array}$} & \multirow{3}{*}{$\begin{array}{c}\begin{array}{c}\% \mathrm{H}_{2} \mathrm{O} \\
\text { Far. }\end{array} \\
0,80^{* *}\end{array}$} & \multirow{2}{*}{\multicolumn{2}{|c|}{\begin{tabular}{l}
\multicolumn{2}{c}{ CNERNA } \\
Vol. $\quad$ NTP
\end{tabular}}} \\
\hline & & & & & & & & & & & & \\
\hline & & & & & & & & & & & $0,89^{* *}$ & $0,84^{* *}$ \\
\hline & W & $0,69^{* *}$ & $0,62^{*}$ & $0,68 * *$ & $-0,46$ & 1 & $0,8 I^{* *}$ & $-0,80^{* *}$ & $-0,14$ & $0,62^{*}$ & $0,60^{*}$ & $0,73^{* *}$ \\
\hline \multirow{2}{*}{ Courtot } & Prot. tot. & 1 & $0,98^{* *}$ & $0,97 * *$ & $-0,26$ & $0,90^{* *}$ & $0,88^{* *}$ & $-0,81^{* *}$ & 0,21 & $0,58^{*}$ & $\begin{array}{l}0,74^{* *} \\
\quad(2)\end{array}$ & $\begin{array}{r}0,17 \\
(2)\end{array}$ \\
\hline & $\mathbf{W}$ & $0,90^{* *}$ & $0,93 * *$ & $0,89^{* *}$ & $-0,32$ & 1 & $0,93^{* *}$ & $-0,82^{* *}$ & 0,33 & 0,52 & $\begin{array}{l}0,75^{* *} \\
(2)\end{array}$ & $\begin{array}{r}0,15 \\
(2)\end{array}$ \\
\hline
\end{tabular}

(1) Les coefficients de corrélations sont calculés sur 15 couples, les échantillons B-N pour « Hardi » et A-R pour « Courtot » ayant été éliminés du fait d'une auantité insuffisante de grains pour le test de panification CNERNA.

(2) Panification en mélange avec 50 p. 100 de farine de «Courtot » et 50 p. 100 de farine standard. 
TABLEAU 4

Essais Inter-stations 1976-77: Variation des tests d'appréciation de la valeur d'utilisation; composantes de cette variation. Inter-station trials : variation in baking quality tests and components of this variation.

\begin{tabular}{|c|c|c|c|c|c|c|c|c|}
\hline $\begin{array}{c}\text { Test/Analyse } \\
\text { (1) }\end{array}$ & $\begin{array}{c}\text { Essai } \\
\text { (2) }\end{array}$ & $\begin{array}{c}\mathrm{Nb} \\
\text { d'échantillons } \\
\text { (3) }\end{array}$ & $\begin{array}{r}\text { Etendue } \\
\text { du } \\
\text { génotypes } \\
\text { (4) }\end{array}$ & $\begin{array}{l}\text { ariation } \\
\text { tère } \\
\text { lieux } \\
(5)\end{array}$ & $\begin{array}{l}\text { p.p.d.s. } \\
\text { génot. } \\
(6)\end{array}$ & $\begin{array}{c}\mathrm{Nb} \text { de } \\
\text { classes } \\
(7)\end{array}$ & $\begin{array}{l}\mathrm{CV} \\
(8)\end{array}$ & $\frac{\sigma_{\mathrm{G}}^{2}}{\sigma_{\mathrm{L}}^{2}}$ \\
\hline Prot. tot. & $\begin{array}{l}\text { PC } 77 \\
\text { PL } 77\end{array}$ & $\begin{array}{l}G=9, L=6 \\
G=13, L=6\end{array}$ & $\begin{array}{l}11,2-12,6 \\
10,4-14,0\end{array}$ & $\begin{array}{l}11,3-12,9 \\
10,9-12,4\end{array}$ & $\begin{array}{l}0,6 \\
0,5\end{array}$ & $\begin{array}{l}2 \\
6,5\end{array}$ & $\begin{array}{l}4,4 \\
4,0\end{array}$ & $\begin{array}{l}0,53 \\
2,41\end{array}$ \\
\hline Zel. & $\begin{array}{l}\text { PC } 77 \\
\text { PL } 77\end{array}$ & $\begin{array}{l}G=10, L=6 \\
G=13, L=6\end{array}$ & $\begin{array}{l}22,2-58,0 \\
14,7-44,7\end{array}$ & $\begin{array}{l}33,1-46,5 \\
26,5-35,6\end{array}$ & $\begin{array}{l}5,2 \\
3,4\end{array}$ & $\begin{array}{l}7 \\
9\end{array}$ & $\begin{array}{r}11,8 \\
9,3\end{array}$ & $\begin{array}{l}5,23 \\
5,07\end{array}$ \\
\hline Pelsh. & $\begin{array}{l}\text { PC } 77 \\
\text { PL } 77\end{array}$ & $\begin{array}{l}G=9, L=6 \\
G=13, L=6\end{array}$ & $\begin{array}{l}41,1-237,7 \\
23,9-280,0\end{array}$ & $\begin{array}{r}119,3-182,8 \\
94,3-146,3\end{array}$ & $\begin{array}{l}21,3 \\
22,9\end{array}$ & $\begin{array}{r}9 \\
11\end{array}$ & $\begin{array}{l}12,2 \\
16,9\end{array}$ & $\begin{array}{r}5,26 \\
18,44\end{array}$ \\
\hline W & $\begin{array}{l}\text { PC } 77 \\
\text { PL } 77\end{array}$ & $\begin{array}{l}\mathrm{G}=9, \mathrm{~L}=6 \\
\mathrm{G}=13, \mathrm{~L}=6\end{array}$ & $\begin{array}{r}117,2-311,0 \\
45,8-246,7\end{array}$ & $\begin{array}{l}175,3-198,9 \\
119,2-156,5\end{array}$ & $\begin{array}{l}26,1 \\
24,7\end{array}$ & $\begin{array}{l}7 \\
8\end{array}$ & $\begin{array}{l}12,0 \\
15,5\end{array}$ & $\begin{array}{r}146,73 \\
27,80\end{array}$ \\
\hline G & $\begin{array}{l}\text { PC } 77 \\
\text { PL } 77\end{array}$ & $\begin{array}{l}G=9, L=6 \\
G=13, L=6\end{array}$ & $\begin{array}{l}17,5-26,9 \\
16,1-27,7\end{array}$ & $\begin{array}{l}22,5-26,5 \\
21,7-26,0\end{array}$ & $\begin{array}{l}1,7 \\
2,1\end{array}$ & $\begin{array}{l}5,5 \\
6\end{array}$ & $\begin{array}{l}6,2 \\
7,6\end{array}$ & $\begin{array}{l}3,89 \\
3,30\end{array}$ \\
\hline $\mathrm{P} / \mathrm{L}$ & $\begin{array}{l}\text { PC } 77 \\
\text { PL } 77\end{array}$ & $\begin{array}{l}G=9, L=6 \\
G=13, L=6\end{array}$ & $\begin{array}{ll}0,25- & 1,32 \\
0,16- & 0,69\end{array}$ & $\begin{array}{l}0,36-0,80 \\
0,28-0,57\end{array}$ & $\begin{array}{l}0,25 \\
0,14\end{array}$ & $\begin{array}{l}4 \\
4\end{array}$ & $\begin{array}{l}35,1 \\
28,3\end{array}$ & $\begin{array}{l}5,69 \\
1,47\end{array}$ \\
\hline$\% \mathrm{H}_{2} \mathrm{O}$ Far. & $\begin{array}{l}\text { PC } 77 \\
\text { PC } 77 \\
\text { PL } 77 \\
\text { PL } 77\end{array}$ & $\begin{array}{l}G=8, L=6 \\
G=9, L=5 \\
G=10, L=6 \\
G=13, L=4\end{array}$ & $\begin{array}{l}25,8-29,6 \\
25,9-29,6 \\
24,4-28,8 \\
24,6-28,4\end{array}$ & $\begin{array}{l}26,9-27,8 \\
27,0-28,0 \\
25,5-26,9 \\
25,5-26,3\end{array}$ & $\begin{array}{l}0,9 \\
0,9 \\
1,0 \\
0,7\end{array}$ & $\begin{array}{l}4 \\
4 \\
4,5 \\
5\end{array}$ & $\begin{array}{l}2,7 \\
2,5 \\
3,2 \\
2,0\end{array}$ & $\begin{array}{r}39,65 \\
17,37 \\
5,50 \\
9,25\end{array}$ \\
\hline Hagb. & $\begin{array}{l}\text { PC } 77 \\
\text { PC } 77 \\
\text { PL } 77\end{array}$ & $\begin{array}{l}G=8, L=6 \\
G=10, L=3 \\
G=11, L=6\end{array}$ & $\begin{array}{l}276,5-355,8 \\
285,0-358,3 \\
244,2-332,5\end{array}$ & $\begin{array}{l}278,1-351,2 \\
301,5-338,5 \\
241,4-322,3\end{array}$ & $\begin{array}{l}39,0 \\
51,6 \\
55,5\end{array}$ & $\begin{array}{l}2 \\
1 \\
2\end{array}$ & $\begin{array}{r}10,4 \\
9,3 \\
17,0\end{array}$ & $\begin{array}{l}0,74 \\
1,77 \\
0,55\end{array}$ \\
\hline Pan. Godon & $\begin{array}{l}\text { PC } 77 \\
\text { PC } 77 \\
\text { PL } 77\end{array}$ & $\begin{array}{l}G=8, L=6 \\
G=9, L=5 \\
G=13, L=4\end{array}$ & $\begin{array}{l}138,3-185,0 \\
135,0-180,0 \\
108,7-191,2\end{array}$ & $\begin{array}{l}136,2-178,9 \\
137,8-177,2 \\
149,6-175,8\end{array}$ & $\begin{array}{l}17,6 \\
19,4 \\
20,7\end{array}$ & $\begin{array}{l}3 \\
2 \\
4\end{array}$ & $\begin{array}{l}9,3 \\
9,5 \\
8,9\end{array}$ & $\begin{array}{l}0,71 \\
0,54 \\
4,09\end{array}$ \\
\hline Pan. Bourdet & $\begin{array}{l}\text { PC } 77 \\
\text { PL } 77\end{array}$ & $\begin{array}{l}G=9, L=5 \\
G=13, L=5\end{array}$ & $\begin{array}{l}430,0-605,0 \\
334,4-649,0\end{array}$ & $\begin{array}{l}500,0-560,0 \\
517,2-564,6\end{array}$ & $\begin{array}{l}66,3 \\
57,7\end{array}$ & $\begin{array}{l}3 \\
5,5\end{array}$ & $\begin{array}{l}9,6 \\
8,4\end{array}$ & $\begin{array}{l}10,17 \\
27,26\end{array}$ \\
\hline Gel. Prot. & $\begin{array}{l}\text { PL } 77 \\
\text { PL } 77\end{array}$ & $\begin{array}{l}G=11, L=5 \\
G=13, L=4\end{array}$ & $\begin{array}{r}4,58-11,52 \\
4,35-11,58\end{array}$ & $\begin{array}{l}6,97-8,17 \\
7,36-8,45\end{array}$ & $\begin{array}{l}0,96 \\
1,06\end{array}$ & $\begin{array}{l}7 \\
7\end{array}$ & $\begin{array}{l}9,8 \\
9,3\end{array}$ & $\begin{array}{l}25,03 \\
24,04\end{array}$ \\
\hline Vol. CNERNA & PC 77 & $\mathrm{G}=6, \mathrm{~L}=4$ & $1256-1500$ & $1337-1450$ & 149 & 2 & 7,1 & 4,61 \\
\hline N.T.P. CNERNA & $\begin{array}{l}\text { PC } 77 \\
\text { PC } 77\end{array}$ & $\begin{array}{l}G=7, L=4 \\
G=6, L=5\end{array}$ & $\begin{array}{l}162,5-208,5 \\
161,0-204,6\end{array}$ & $\begin{array}{l}160,9-206,9 \\
156,8-206,7\end{array}$ & $\begin{array}{l}45,4 \\
32,1\end{array}$ & $\begin{array}{l}1 \\
1\end{array}$ & $\begin{array}{l}16,4 \\
13,1\end{array}$ & $\begin{array}{l}0,21 \\
0,40\end{array}$ \\
\hline Rdt & $\begin{array}{l}\text { PC } 77 \\
\text { PL } 77\end{array}$ & $\begin{array}{l}G=9, L=6 \\
G=13, L=6\end{array}$ & $\begin{array}{l}56,4-66,5 \\
53,0-64,6\end{array}$ & $\begin{array}{l}43,9-70,0 \\
40,9-72,0\end{array}$ & $\begin{array}{l}7,8 \\
5,4\end{array}$ & $\begin{array}{l}1 \\
2\end{array}$ & $\begin{array}{r}11,0 \\
7,9\end{array}$ & $\begin{array}{l}0,04 \\
0,09\end{array}$ \\
\hline
\end{tabular}

(2) Nous avons autant que possible retenu les mêmes génotypes et les mêmes lieux pour chacun des tests afin de permettre une comparaison rigoureuse entre eux. Cependant, il peut arriver que certains effectifs soient différents du fait de données manquantes.

$\mathrm{Si}$ on considère les données présentées dans le tableau 4 et plus particulièrement celles des colonnes 7,8 et 9 , en faisant abstraction de la signification «technologique» des tests pratiqués, les tests ou analyses les plus intéressants pour cribler des génotypes en cours de sélection, c'est-à-dire ceux permettant d'établir un grand nombre de classes de « qualité » et présentant un rapport $\frac{\sigma_{G}^{2}}{\sigma_{I}^{2}}$ élevé, sont : l'indice de sédimentation de Zeleny, le test de Pelshenke, le $\mathrm{W}$ de l'alvéographe Chopin et le gel protéique (épaisseur).

Ainsi, les essais de cuisson (tests plus synthétiques) ne répondent qu'imparfaitement aux critères de choix énumérés. Cependant, le test de micro-cuisson de BOURDET et al. (1973), peu influencé par le milieu et permettant d'établir 5 classes de qualité dans le cas de l'échantillonnage des génotypes de "paille longue », est susceptible de retenir l'intérêt des sélectionneurs.

Ces résultats sont, à quelques détails près, en accord avec les conclusions exposées par ROUSSET \& LOISEL (1984) concernant des essais réalisés lors d'une autre campagne (1977-78) sur un échantillonnage génotypique différent.

Les résultats des corrélations intergénotypiques et intra-lieux présentés dans les tableaux 5 et 6 nous révèlent un comportement des tests bien différents de celui observé dans le cas de l'essai "Agronomie".

La prise en compte d'une variation génétique « à lieu constant " (tabl. 5) montre que la liaison entre les résultats des différents tests et la teneur en protéines totales est faible. Le niveau parfois élevé des corrélations négatives entre la teneur en protéines totales et le 
TABLEAU 5

Essais inter-stations 1976-77 - Coefficients de corrélation de la teneur en protéines totales avec chacun des autres tests. Inter-station trials 1976-77-Correlation coefficients between protein content and every other test.

\begin{tabular}{|c|c|c|c|c|c|c|c|c|c|c|}
\hline & \multirow[b]{2}{*}{ Zel. } & \multirow[b]{2}{*}{ Pelsh. } & \multirow[b]{2}{*}{ W } & \multirow[b]{2}{*}{$\mathrm{G}$} & \multirow[b]{2}{*}{$\mathrm{P} / \mathrm{L}$} & \multirow[b]{2}{*}{ Hagb. } & \multirow[b]{2}{*}{$\begin{array}{c}\% \mathrm{H}_{2} \mathrm{O} \\
\text { Far. }\end{array}$} & \multicolumn{2}{|c|}{$\begin{array}{l}\text { Volume du pain } \\
\text { en panification }\end{array}$} & \multirow[b]{2}{*}{ Rdt } \\
\hline & & & & & & & & Godon & Bourdet & \\
\hline Clermont (1) & 0,30 & 0,00 & 0,27 & $0,38^{* *}$ & $-0,28$ & $0,43^{*}$ & 0,20 & 0,05 & $0,41^{*}$ & $-0,61 * *$ \\
\hline Colmar (1) & 0,21 & $-0,08$ & 0,05 & 0,27 & $-0,19$ & $-0,09$ & $-0,01$ & $-0,32$ & 0,10 & $-0,68^{* *}$ \\
\hline Dijon (1) & 0,14 & $-0,24$ & 0,10 & 0,33 & $-0,22$ & 0,06 & $-0,07$ & 0,07 & 0,18 & $-0,71 * *$ \\
\hline La Minière (1) & 0,25 & $-0,10$ & 0,23 & 0,41 & $-0,15$ & 0,05 & 0,14 & $-0,01$ & $-0,06$ & $-0,30$ \\
\hline Mons (1) & $0,36^{*}$ & $-0,01$ & 0,13 & $0,44^{*}$ & $-0,29$ & $-0,18$ & 0,14 & $0,37^{*}$ & $0,35^{*}$ & 0,05 \\
\hline Rennes (1) & $0,61 * *$ & 0,21 & $0,42^{*}$ & $0,50^{* *}$ & $-0,25$ & 0,30 & $0,55^{* *}$ & 0,23 & 0,26 & $-0,45^{*}$ \\
\hline $\begin{array}{l}\text { Globale (2) } \\
\text { (paille longue) }\end{array}$ & $0,51^{*}$ & $-0,07$ & 0,15 & 0,45 & $-0,37$ & $-0,21$ & 0,35 & $-0,01$ & 0,11 & $-0,42$ \\
\hline $\begin{array}{l}\text { Globale (3) } \\
\quad \text { (paille courte) }\end{array}$ & 0,42 & $-0,03$ & 0,17 & 0,27 & $-0,16$ & 0,20 & 0,35 & $-0,37$ & $-0,16$ & $-0,56^{*}$ \\
\hline
\end{tabular}

Les résultats de l'essai de panification CNERNA réalisé uniquement sur la série « paille courte » sur 7 génotypes ne peuvent figurer dans les tableaux 5 et 6.

(1) Le calcul des corrélations intergénotypiques intra-lieux regroupe les résultats des 2 séries " paille longue " et " paille courte ". 23 variétés et 23 échantillons.

(2) et (3) Les corrélations globales prennent en compte les moyennes génotypiques sur l'ensemble des lieux en considérant séparément les 2 séries paille longue et paille courte.

(2) $=13$ variétés et 78 échantillons.

(3) $=10$ variétés et 60 échantillons.

TABLEAU 6

Essais inter-stations 1976-77 - Coefficients de corrélation entre $W$ de l'alvéographe et chacun des autres tests. Inter-station trials 1976-77-Correlation coefficients between $W$ alveograph and every other test.

\begin{tabular}{|c|c|c|c|c|c|c|c|c|c|c|c|}
\hline & & \multirow[b]{2}{*}{$\begin{array}{l}\text { Prot. } \\
\text { tot. }\end{array}$} & \multirow[b]{2}{*}{ Zel. } & \multirow[b]{2}{*}{ Pelsh. } & \multirow[b]{2}{*}{$\mathrm{G}$} & \multirow[b]{2}{*}{$\mathrm{P} / \mathrm{L}$} & \multirow[b]{2}{*}{ Hagb. } & \multirow[b]{2}{*}{$\mathrm{H}_{2} \mathrm{O}$ Far. } & \multicolumn{2}{|c|}{$\begin{array}{l}\text { Volume du pain } \\
\text { en panification }\end{array}$} & \multirow[b]{2}{*}{ Rdt } \\
\hline & & & & & & & & & Godon & Bourdet & \\
\hline Clermont (1) & & 0,27 & $0,89^{* *}$ & $0,78^{* *}$ & 0,23 & 0,21 & $0,65^{* *}$ & $0,60^{* *}$ & 0,33 & $0,58^{* *}$ & $-0,09$ \\
\hline Colmar (1) & & 0,05 & $0,90^{* *}$ & $0,79^{* *}$ & $-0,06$ & $0,45^{*}$ & $0,70^{* *}$ & $0,76^{* *}$ & $0,53^{* *}$ & $0,49 * *$ & 0,33 \\
\hline Dijon (1) & & 0,10 & $0,90^{* *}$ & $0,82^{* *}$ & 0,34 & 0,13 & $0,52^{* *}$ & $0,51^{* *}$ & $0,80^{* *}$ & $0,72^{* *}$ & 0,10 \\
\hline La Minière (1) & & 0,23 & $0,88^{* *}$ & $0,82 * *$ & 0,28 & 0,28 & $0,50^{* *}$ & $0,52^{* *}$ & $0,50^{* *}$ & $0,42 *$ & 0,32 \\
\hline Mons (1) & & 0,13 & $0,88^{* *}$ & $0,87^{* *}$ & 0,26 & $0,38^{*}$ & $0,51^{* *}$ & $0,69 * *$ & 0,23 & $0,62^{* *}$ & 0,29 \\
\hline Rennes (1) & & 0,42 & $0,87^{* *}$ & $0,92 * *$ & $0,42^{*}$ & 0,05 & $0,52^{* *}$ & $0,67 * *$ & $0,46^{*}$ & $0,47^{*}$ & $-0,34$ \\
\hline $\begin{array}{l}\text { Globale (2) } \\
\quad \text { (paille longue) }\end{array}$ & & 0,15 & $0,78^{* *}$ & $0,87^{* *}$ & 0,43 & 0,00 & 0,31 & 0,36 & $0,71^{* *}$ & $0,54 *$ & 0,08 \\
\hline $\begin{array}{l}\text { Globale (3) } \\
\quad \text { (paille courte) }\end{array}$ & & 0,17 & $0,84^{* *}$ & $0,71^{* *}$ & $-0,18$ & 0,41 & $0,55^{*}$ & $0,77^{* *}$ & 0,27 & 0,47 & $-0,04$ \\
\hline
\end{tabular}

(1), (2) et (3) voir tableau 5.

rendement en grain rappelle la nécessité d'une fumure azotée suffisante pour concilier un haut niveau de rendement avec une teneur élevée en protéines.

En ce qui concerne la liaison entre les résultats des différents tests et le $\mathrm{W}$ de l'alvéographe Chopin pris comme test de référence (tabl. 6), on constate que l'indice de sédimentation de Zeleny et le test de Pelshenke sont fortement reliés au $\mathrm{W}$ et ce, régulièrement sur l'ensemble des lieux. On observe par ailleurs des liaisons en général significatives voire hautement significatives pour le test de Hagberg, le taux d'hydratation au farinographe Brabender et surtout les essais de cuisson miniaturisée et de micro-cuisson.

Dans le cas présent, le rendement en grain n'est jamais lié significativement au $\mathrm{W}$, ce qui confirme la possibilité de concilier rendement et valeur d'utilisation élevés (en ne considérant pas la catégorie des "blés de force " nécessairement riches en protéines).

\section{DISCUSSION-CONCLUSION}

Le but de cet article n'est pas d'expliquer les bases biochimiques de la variation des tests de valeur d'utilisation en fonction des différentes sources de cette variation, il est essentiellement de montrer que, suivant l'origine de la variation, les liaisons entre les tests peuvent être différentes. On a vu, en particulier, que suivant le test et suivant le mode d'obtention des échantillons, on pouvait conférer au facteur « teneur en protéines totales » un rôle explicatif de la variation des résultats plus ou moins important. Il arrive, dans le cas où un facteur du milieu agit directement sur l'accumulation des protéines dans le grain, que la variation d'un test particulier soit explicable en grande partie par la variation quantitative des protéines. Ceci n'est pas nécessairement vrai lorsque l'on s'adresse à une variation génétique de la valeur d'utilisation. 
Ces résultats ne font que confirmer les observations d'un grand nombre d'auteurs sur la liaison pouvant exister entre une variation quantitative des protéines et de la variation de l'expression de certains tests. Ainsi pour l'indice de sédimentation de Zeleny, ZELENY lui-même (1947), MESDAG (1964), ROUSSET \& AUTRAN (1977) signalent cette liaison ; pour l'essai de panification CNERNA, ROUSSET \& AUTRAN (1977) indiquent une relation curvilinéaire entre la note totale en panification et la teneur en protéines totales. A la suite de telles observations, les mêmes auteurs avaient proposé différents modes de transformation ou d'interprétation des résultats du test de Zeleny pour une meilleure approche de la valeur d'utilisation génotypique.

Les relations étroites observées dans le cas de l'essai «Agronomie » ne se retrouveront pas nécessairement pour des séries d'échantillons obtenus pour un même génotype dans des conditions d'environnement très diverses (différents lieux, différentes années...) et pour lesquels une gamme très large de teneurs en protéines totales résulterait de facteurs «limitants » du milieu variés, comme par exemple la fumure azotée, l'alimentation hydrique, les attaques parasitaires... Dans de telles conditions, une même modification quantitative des protéines peut s'accompagner d'une autre variation qualitative des protéines et se traduire par une variation différente des critères de valeur d'utilisation.

A côté du comportement assez clair de la plupart des tests étudiés dont la variation a pu être expliquée directement par la variation quantitative des protéines, le test de Pelshenke a révélé un comportement particulier et son expression est apparue peu ou non influencée par la teneur en protéines totales. Cette particularité ne devra pas être oubliée comme critère de choix parmi les tests lorsqu'il s'agira de définir les méthodes d'appréciation de la valeur d'utilisation d'un lot de grains.

Les résultats et conclusions de l'étude analytique de la variation de la valeur d'utilisation sous l'influence des facteurs du milieu (essai "Agronomie ») sont fondamentalement différents de ceux qu'a révélé l'étude de la variation génétique de la valeur d'utilisation (essais « inter-stations »). L'étude des relations entre les différents tests d'appréciation de la valeur d'utilisation nous a conduits au calcul de corrélations «environnementales» (intra-génotypiques, interenvironnementales) dans le cas de l'essai «Agronomie » et de corrélations «phénotypiques » (intergénotypiques, intra-lieux) dans le cas de l'essai « interstations $"$.

Dans le $1^{\text {er }}$ cas, le coefficient de corrélation élevé obtenu par exemple entre l'indice de sédimentation de Zeleny et le $\mathrm{W}$ de l'alvéographe Chopin pour «Courtot " ne fait que traduire le degré de liaison élevé de chacun de ces tests avec la teneur en protéines totales. Par contre, les coefficients de corrélation faibles voire négatifs observés entre l'indice Pelshenke et les autres tests résultent du comportement particulier de ce test comparé aux autres vis-à-vis de la teneur en protéines totales. Une conclusion hâtive pourrait conduire à éliminer ce test dans l'appréciation de la valeur génotypique d'utilisation.

Dans le $2^{\mathrm{e}}$ cas (essais inter-stations) par contre, la plupart des tests sont corrélés positivement et en général significativement. En particulier l'indice de Pelshenke est alors étroitement corrélé au W de l'alvéographe Chopin, test de référence dans l'évaluation de la force boulangère. Ceci contribue à confirmer le choix de ce test, compte tenu de sa simplicité, pour l'appréciation de la valeur génotypique d'utilisation dans les générations précoces de sélection. Les corrélations phénotypiques observées résulteraient de variations qualitatives de ces protéines.

A la lumière des études que nous avons conduites et de certains résultats bibliographiques, il apparaît que le degré de liaison des tests pratiqués avec la teneur en protéines totales, en particulier dans le cas des composantes de l'alvéogramme Chopin, des indices de sédimentation Zeleny et SDS, du volume du pain dans les essais de panification..., pourrait constituer une indication assez précise de l'influence d'un ou de plusieurs facteurs du milieu sur l'expression de la valeur d'utilisation, influence à laquelle le sélectionneur s'attache à soustraire le matériel végétal dont il souhaite apprécier la valeur génotypique. C'est pourquoi, du point de vue du sélectionneur, les études comparatives entre critères d'appréciation de la valeur d'utilisation du grain de blé les plus dignes de confiance seraient celles pour lesquelles la variation quantitative des protéines intervient peu (de manière non significative) dans l'expression de tous les tests pratiqués, à condition par ailleurs que la gamme de variation des échantillons retenus dans l'étude corresponde à celle couramment rencontrée dans le matériel végétal soumis à la sélection.

La réflexion conduite dans notre développement concernant l'influence relative du génotype et de l'environnement sur l'expression de la valeur d'utilisation ainsi que le rôle essentiel joué par la quantité de protéines totales rejoint celle de JEANJEAN \& FEILLET (1977) dans leur tentative d'approche de la «qualité intrinsèque d'une variété " indépendante des conditions de croissance de la plante. Ces auteurs distinguent le test de "sélection », qui doit rendre compte de la qualité intrinsèque des génotypes, du test « commercial », qui rend compte de l'interaction qualité intrinsèque $\times$ conditions de croissance de la plante et dont l'expression sera très influencée par la teneur en protéines. Cette réflexion et les travaux expérimentaux s'y rapportant les conduisent à proposer la teneur en "gel protéique » comme test de sélection répondant aux critères énoncés.

Les mêmes résultats que ceux que nous avons présentés (essai «Agronomie ») ou des résultats obtenus dans des essais analogues (essai inter-stations) analysés par ROUSSET \& LOISEL (1984) ont permis à ces auteurs d'indiquer des éléments du choix des différents tests en fonction de la nature des échantillons (et de leur variation) auxquels on s'adresse.

Les observations présentées dans cet article nous conduisent à mettre l'accent sur le soin particulier qui doit être apporté au choix des échantillons et à la description précise de leurs conditions d'obtention lorsque l'on veut conduire des études comparatives entre tests d'appréciation de la valeur d'utilisation. C'est le cas lorsque l'on souhaite faire l'étude critique d'un nouveau test. On a pu en effet constater, au cours d'analyses bibliographiques, de grandes discordances 
entre résultats de corrélations entre tests. On peut supposer que de telles discordances tiennent à la fois aux caractéristiques de distribution des échantillons analysés (moyenne, amplitude de variation...) et à l'origine de la variation observée (génotype, environnement, interaction génotype $x$ environnement).

Enfin, à la lumière des résultats de nos expérimentations, il nous semble qu'un choix judicieux d'échantillons devrait permettre d'aider à la connaissance des bases biochimiques de la valeur d'utilisation du blé tendre dans les industries de cuisson.

Recu le 28 mars 1984 Accepté le 21 mars 1985.

\section{REMERCIEMENTS}

Les auteurs remercient les personnes qui ont participé aux expérimentations qui sont rapportées dans cet article pour la réalisation des essais, la collecte des échantillons et leur analyse.

Ils remercient également Messieurs P. Auriau, G. Doussinault et $\mathrm{F}$. KAAN qui ont bien voulu apporter une correction constructive à ce texte.

\section{RÉFÉRENCES BIBLIOGRAPHIQUES}

Axford D. W. E., Mc Dermotl E. E., Redman D. G.. 1978. Smallscale tests of bread making quality. Milling Feed and Fertilizer, 161 (5), $18-20$.

Axford D. W. E., Mc Dermott E. E., Redman D. G., 1979. Note on the Sodium Dodecyl Sulfate test of breadmaking quality : comparison with Pelshenke and Zeleny tests. Cereal Chemistry, 56 (6), 582-584.

Bourdet A., Berrier R., Leclercq J. R., 1971. Signification technologique des critères dont dispose la sélection pour apprécier l'aptitude boulangère des variétés nouvelles. Meunerie française, 275-276, $1-22$.

Bourdet A., Berrier R., 1973. Micro-essai de cuisson pour différencier les variétés de blé tendre selon leur force. C.R. Acad. Agric. Fr., 59 (1), 59-65.

Bourdet A., Berrier R., Autran J. C., 1973. Essai de cuisson miniaturisé pour apprécier la force des blés tendres. Meunerie française, 295, 11-14.

Branlard G., Triboi E., 1983. Influence du milieu de culture sur les concentrations relatives des bandes des gliadines chez le blé tendre. C.R. Acad. Sci. Paris, 297, série III, 229-232.

Gachon L., 1973. Influence du système cultural sur l'économie de l'azote en sol calcaire de Limagne. Sci. Sol, 3, 177-197.

Gachon L., 1975. Participation des légumineuses au bilan de l'azote en sol argilo-calcaire de Limagne. Sci. Sol, 2, 89-100.
Godon B., Sarazin J., 1973. Signification d'un test de panification à échelle réduite. Ann. Technol. Agric., 22 (1), 21-33.

Jeanjean M. F., Feillet P., 1977. Relation entre la teneur en « gel protéique » et la qualité technologique des blés. Coll. "Utilisation des acquisitions de la biochimie pour la sélection des graines de céréales pour leur qualité technologique ». Odessa, novembre 1977, document ronéotypé, $25 \mathrm{p}$.

Mauze C., Richard M., Scotti G., 1972. Guide pratique. Contrôle de la qualité des blés. Institut Technique des Céréales et des Fourrages, $176 \mathrm{p}$.

Mesdag J., 1964. Variations in the protein content of wheat and its influence on the sedimentation value and the baking quality. Euphytica, 13, 250-261.

Rousset M., Autran J. C., 1977. La qualité des blés. Coll. « Le Pain ", Paris, 14 et 15 novembre 1977. CNRS Ann. Nutr. Aliment., 15-42.

Rousset M., Loisel W., 1984. Les tests d'appréciation de la valeur d'utilisation du blé tendre dans les industries de cuisson, 467-509. In "Guide pratique d'analyses dans les industries des céréales". APRIA, Lavoisier, $685 \mathrm{p}$.

Zeleny L., 1947. A simple sedimentation test for estimating the bread making and gluten qualities of wheat flour. Cereal Chem., $24,465-475$. 